\title{
Efeito dos alfa-bloqueadores no tratamento da ureterolitíase distal - uma revisão sistemática e meta-análise
}

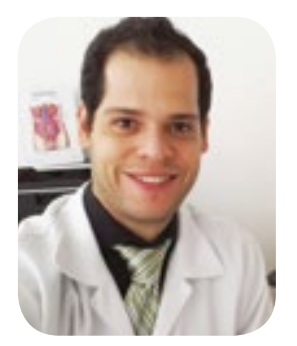

\section{RESUMO}

INTRODUÇÃO: o uso de alfa-bloqueadores no contexto da terapia médica expulsiva para cálculos ureterais tem sido opção importante para manejo de pacientes com dor lombar não complicada. O objetivo deste trabalho é avaliar a eficácia da terapia expulsiva com alfa-bloqueadores para cálculos ureterais distais e resumir as melhores evidências em um algoritmo geral de orientação. MÉTODO: revisão sistemática foi realizada em fevereiro de 2018, no banco de dados do PubMed/ MedLine, com estratégia de busca avançada para seleção de ensaios clínicos randomizados sobre o uso dos alfa-bloqueadores no manejo de paciente com ureterolitíase distal. Os dados foram compilados e meta-analisados em relação à taxa de eliminação e de acordo com o tamanho do cálculo. RESULTADOS: foram selecionados 29 estudos com dados de 6.216 pacientes. A taxa geral de eliminação foi maior com uso de alfa-bloqueador (OR=3,05; IC95\% 2,38-3,91; $p<0,0001$ ), comparativamente à terapia padrão. $O$ efeito se deveu basicamente aos cálculos > 5mm (OR=2,33; IC95\% 1,92 a 2,83; $p<0,0001$ ), não tendo evidenciado efeito significativo para cálculos $<5 \mathrm{~mm}(\mathrm{RR}=1,14$; IC95\% 0,99 a 1,31; $\mathrm{p}=0,0505$ ). Alfa-bloqueador também reduziu o tempo de eliminação em pouco mais de 2 dias (MD= -2,32; IC95\% $-3,40$ a $-1,24 ; p<0,0001)$, sem aumentar a quantidade relatada de efeitos adversos ( $R R=1,27 ; \mathrm{IC} 95 \% 0,98$ a $1,66 ; p=0,065)$. CONCLUSÃO: o uso de alfa-bloqueador é eficaz e seguro no contexto da terapia expulsiva e está indicado para cálculos ureterais distais entre 5 e $10 \mathrm{~mm}$ de diâmetro.

PALAVRAS-CHAVE: alfa-bloqueador, terapia médica expulsiva, cálculo ureteral distal.

KEY-WORDS: alpha blocker, medical expulsive therapy, distal ureteral stone.
Leandro Frarlley Santos e Miranda ${ }^{1}$

\section{INTRODUÇÃO}

A urolitíase é um problema comum dentro da urologia e uma das grandes razões para procurar um especialista. Estima-se que cerca de 5 a $10 \%$ da população sofrem dessa doença ${ }^{1}$. A incidência e prevalência dos cálculos urinários podem ser afetadas por fatores genéticos, nutricionais e ambientais. Caucasianos parecem sofrer mais de urolitíase quando comparados a asiáticos, hispânicos e afro-americanos ${ }^{2}$.

Dentro da história natural, alguns cálculos podem migrar e passar espontaneamente via urinária abaixo. É durante a migração ureteral da pedra que a maioria dos cálculos torna-se sintomática, fazendo o paciente procurar o setor de emergência. O sucesso na eliminação espontânea está associado a fatores como tamanho do cálculo e anatomia do paciente. A posição distal do cálculo ureteral no momento do diagnóstico passa assim a ser um fator prognóstico importante ${ }^{3}$.

Estudos observacionais prévios mostram que, para cálculos ureterais $<5 \mathrm{~mm}$, a taxa de eliminação espontânea é de $68 \%$, reduzindo para $47 \%$, quando entre $5-10 \mathrm{~mm}^{4}$. Quando o cálculo é distal, a taxa de eliminação espontânea pode chegar a $75 \%$, se $<10 \mathrm{~mm}^{2,4}$.

Atualmente, grande parte dos cálculos ureterais, principalmente aqueles $<1 \mathrm{~cm}$, é tratada clinicamente na ausência de complicações ${ }^{4}$. Muitos ensaios clínicos pequenos já foram publicados, mostrando a eficácia dos alfa-bloqueadores (Tansulosina, Doxazosina, Alfuzosina e Naftopidil) com incremento da taxa de eliminação de cálculos, levando à fixação do termo, já bastante utilizado na sala de emergência, Terapia Médica Expulsiva (TME). No nosso meio, duas das drogas mais utilizadas nesse cenário são a Tansulosina e a Doxazosina. Revisões desses trabalhos têm confirmado o efeito benéfico dessas drogas, principalmente em pacientes com cálculo distal, sendo uma das últimas publicadas em 2014 pela colaboração Cochrane $e^{5,6}$. 
Após 4 anos da revisão Cochrane, alguns ensaios recentes e maiores, como o SUSPEND Trial (2015), o estudo de Meltzer (2017) e o estudo de Ye (2017), trouxeram novas, melhores e, por vezes, controversas evidências que ainda não foram analisadas em conjunto com as anteriores. Dessa forma, a proposta deste trabalho é trazer de forma sistematica as melhores evidências da literatura a respeito do efeito dos dois principais alfa-bloqueadores (Tansulosina e Doxazosina) na terapia expulsiva dos cálculos ureterais distais e propor um algoritmo de condução do paciente na sala de emergência.

\section{MÉTODO}

Em fevereiro de 2018 foi realizada revisão de literatura no banco de dados do PubMed/MedLine e seleção passo a passo dos artigos, como sugerida pelo método PRISMA', sem limitação por data de publicação. Para isso, foram utilizadas as palavras-chave "ureterolithiasis" e "alpha-blocker" como a seguinte estratégia: (ureterolithiasis [MeSHTerms]) OR alpha-blocker [MeSHTer$\mathrm{ms}$. Os resultados desta busca inicial foram filtrados posteriormente para mostrar apenas os "Clinical Trials". Inicialmente, os estudos foram selecionados com base na leitura dos títulos e dos abstracts. As referências de todos os trabalhos inicialmente selecionados foram utilizadas para aquisição de estudos adicionais. A seleção final dos trabalhos foi lida na íntegra.

Critérios de Inclusão e Exclusão:

Foram incluidos os ensaios clínicos em adultos, como cálculo ureteral distal de até $10 \mathrm{~mm}$, com pelo menos um grupo de tratamento usando Tansulosina ou Doxazosina isoladamente, comparado com um grupocontrole (placebo ou tratamento padrão).

studos com cálculos não distais, cálculos $>10 \mathrm{~mm}$ na amostra, população não adulta, comparação ou estudos com outras drogas e população heterogênea (pacientes com pielonefrite, cálculos múltiplos, cálculos bilaterais, rim único, malformação do trato urinário ou uso de litotripsia extracorpórea (LECO) ou qualquer outro tratamento concomitante) foram excluídos.

Padronização dos Grupos de Intervenção e Controle Para fins analíticos, os estudos foram divididos segundo o grupo de intervenção em Tansulosina ou Doxazosina, e seus efeitos foram analisados em conjunto (sob o título de alfa-bloqueadores) ou separadamente. Adicionalmente, análise de subgrupo foi realizada quando possivel, dividindo o efeito do tratamento de acordo com o tamanho dos calculos ( $<5 \mathrm{~mm}$ ou $>5 \mathrm{~mm}$ ).

Para o grupo-controle, foi permitido uso apenas de placebo e/ou analgesia, descrito como tratamento padrão (antiespasmódicos, AINE e/ou opioides com ou sem hidratação)

\section{Seleção dos Desfecho}

Os seguintes desfechos foram selecionados para avaliar a eficácia e segurança da TME: taxa de eliminação do cálculo ao fim do tratamento, tempo para eliminação, necessidade de analgésicos (medida pelo uso médio em mg de diclofenaco por dia) e número de efeitos adversos do tratamento, todos analisados em relação ao grupo placebo ou terapia padrão.

Análise Qualitativa dos Estudos

Os estudos foram estratificados qualitativamente, segundo a escala de Jadad (1996) para ensaios clínicos randomizados ${ }^{8}$.

Análise Quantitativa dos Estudos

Foi utilizada a odds-ratio(OR) ou o RiskRatio(RR) para medida sumária do tamanho do efeito para as variâveis dicotômicas e a diferença entre as médias (MD) para as variáveis contínuas. Quando disponíveis apenas a mediana e a amplitude dos parâmetros considerados, as estimativas da média e dos desvios-padrão foram calculadas utilizando o método descrito por $\mathrm{Hozo}^{\circ}$. Para cálculo das estimativas dos efeitos combinados, foi utilizado o método de Cochrane-Mantel-Haenszel para as variáveis dicotômicas e o método de Hedges para as contínuas com os modelos de efeito fixo ou randômico, de acordo com a ausência ou presença de heterogeneidade significativa entre os estudos. Medida de inconsistência (12) foi utilizada para avaliar a variabilidade entre os tamanhos de efeito decorrente de heterogeneidade estatística não explicada por erro aleatório.

As diferenças de tamanho do efeito combinado entre os grupos foram testadas com a estatística de teste " $z$ ", tomando $p<0,05$ como significativo.

Foi realizada análise para potencial presença de viés de publicação, utilizando o método de correlação de Begg-Mazumdar, quando necessario, e exibida atravès de gráficos padrões do tipo funell-plot.

A análise dos dados e confecção dos gráficos foram realizadas com o uso do software R (Version 3.4.0) sobre a plataforma RStudio $\circledast$ (Version 1.0.143 - () 2009 2016 RStudio, Inc.).

\section{RESULTADOS}

Revisão da Literatura

Após aplicação dos criterios descritos, foram sele- cionados 29 estudos, totalizando 6.216 pacientes ( $\mathrm{Fi}$ ura 1) Dos estudos de Yilmaz (2005) e Lojanapiwat (2008) foram extraídos os dados separadamente dos grupos tansulosina e doxazosina, totalizando 31 unidades comparativas para meta-análise.

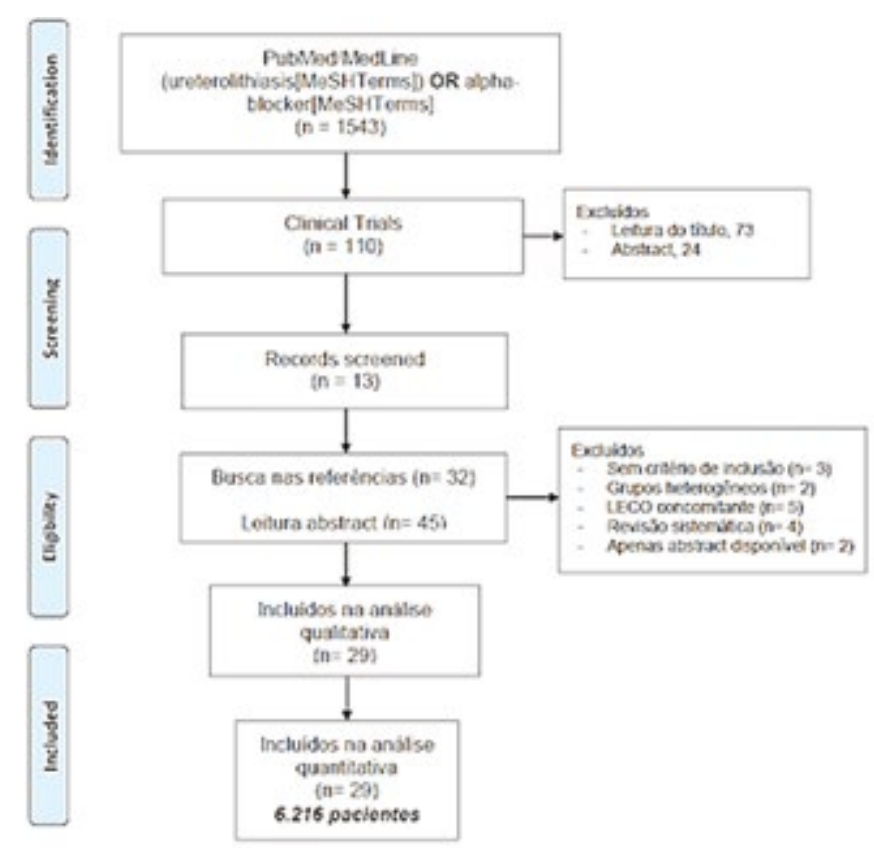

Figura 1 - Fluxograma PRISMA ${ }^{7}$

Análise Qualitativa

A Tabela 1 mostra as características sumárias de cada estudo e a Figura 2 mostra a avaliação para o risco de viés de acordo com escala de Jadad (2008)8.

Análise Quantitativa

Caracteristicas dos grupos:

Não houve diferença significativa entre os grupos tratamento e controle na combinação de estudos de acordo com idade (MD= 0,026, IC $\mathrm{p}=0,96)$, número de homens e mulheres $(\mathrm{OR}=0,92$ IC95\% 0,73 a 1,16; $p=0,49)$ e tamanho do cálculo (MD= 0,$02 ; \mathrm{IC} 95 \%-0,08$ a 0,$13 ; p=0,63$ )

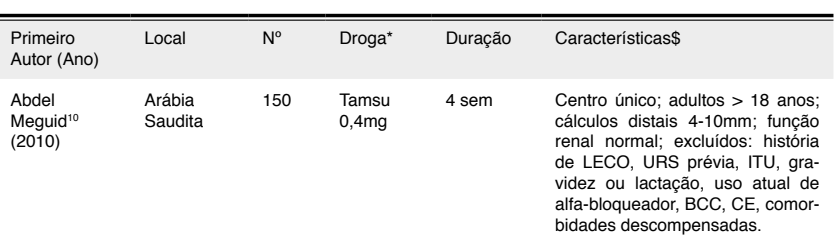

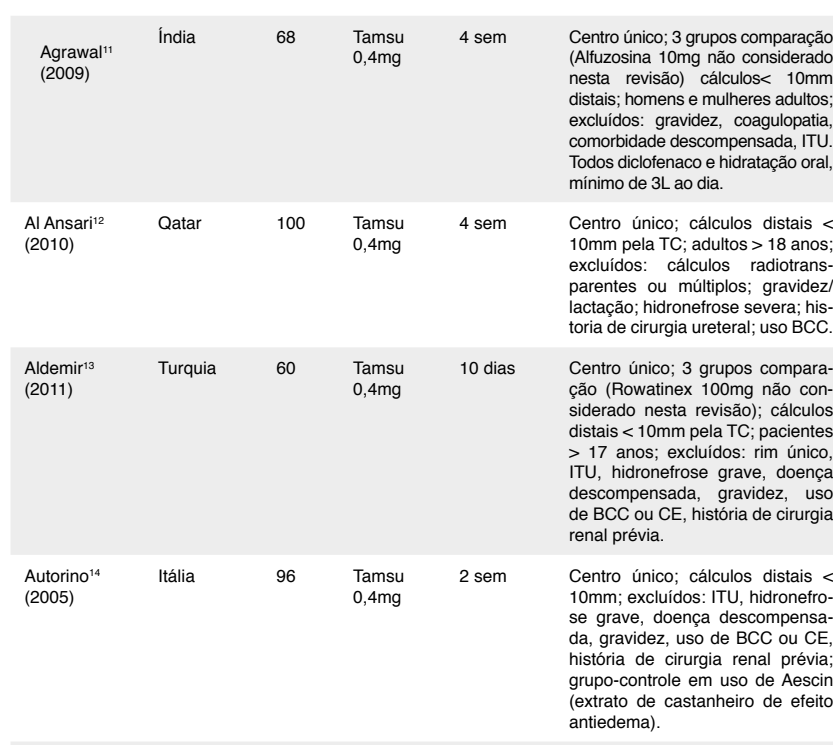

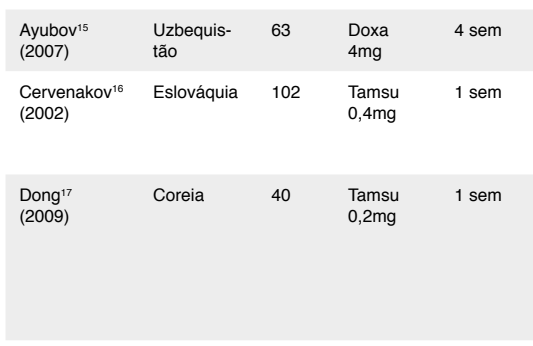

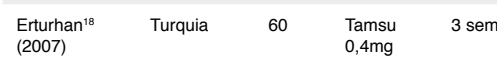

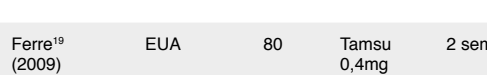

Hen" (2005) $\quad$ Coreia $\quad 67 \quad \underset{\substack{\text { Tamsu } \\ 0.299}}{4}$

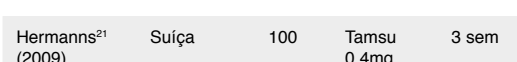

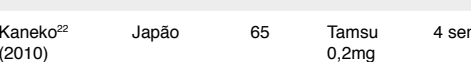

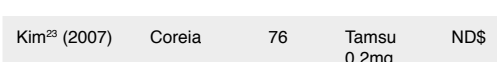

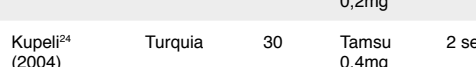

$\begin{array}{lllll}\begin{array}{l}\text { Liatsikos } \\ (2007)\end{array} & \text { Grécia } & 73 & \begin{array}{c}\text { Doxa } \\ 4 m g\end{array} & 4 \text { sem } \\ 4 m g & & \end{array}$

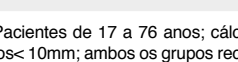

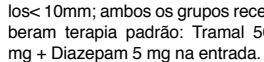

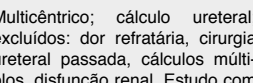

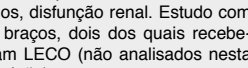

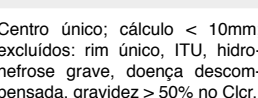

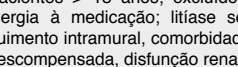

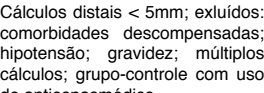

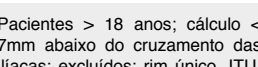

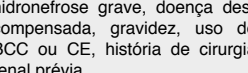

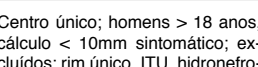

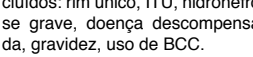

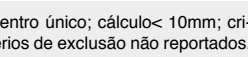

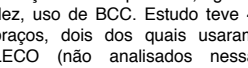

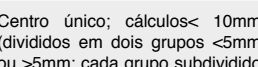

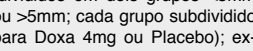
eg grave, doenca descompens

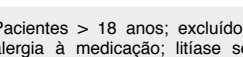

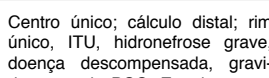




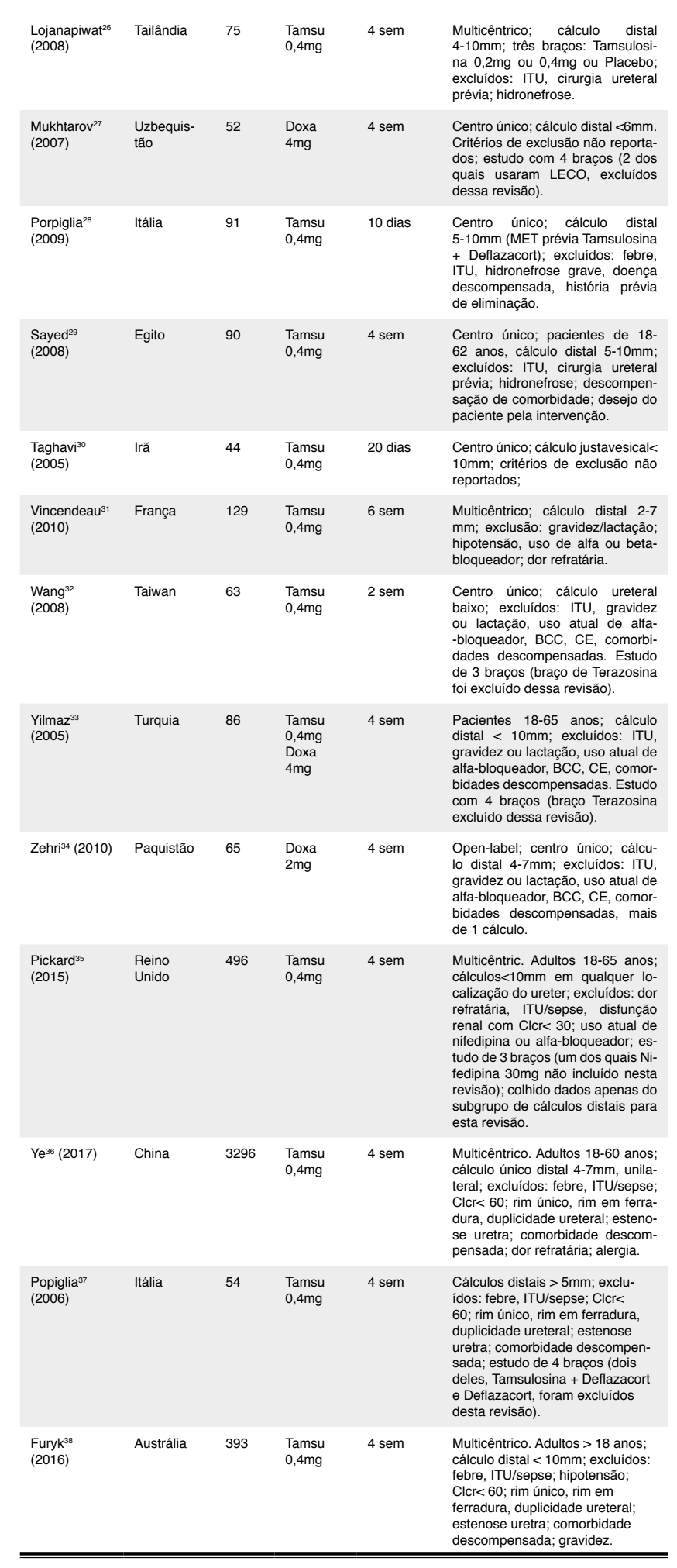

§Número da referênncia em sobrescrito; todos os estudos são Ensaios Clinicos SNámero da reft
Randomizados.

"Tamsu= Tansulosina; Doxa= Doxasozin
\$LECO, litotripsia extracoropórea por ondas de choque; URS, ureterorrenosco-
pia; ITU, infecção de trato urinário; BCC, bloqueador de canais de cálcio; CE, corticosteroides; TC, tomografia computadorizada; ND, nãa disponivivel

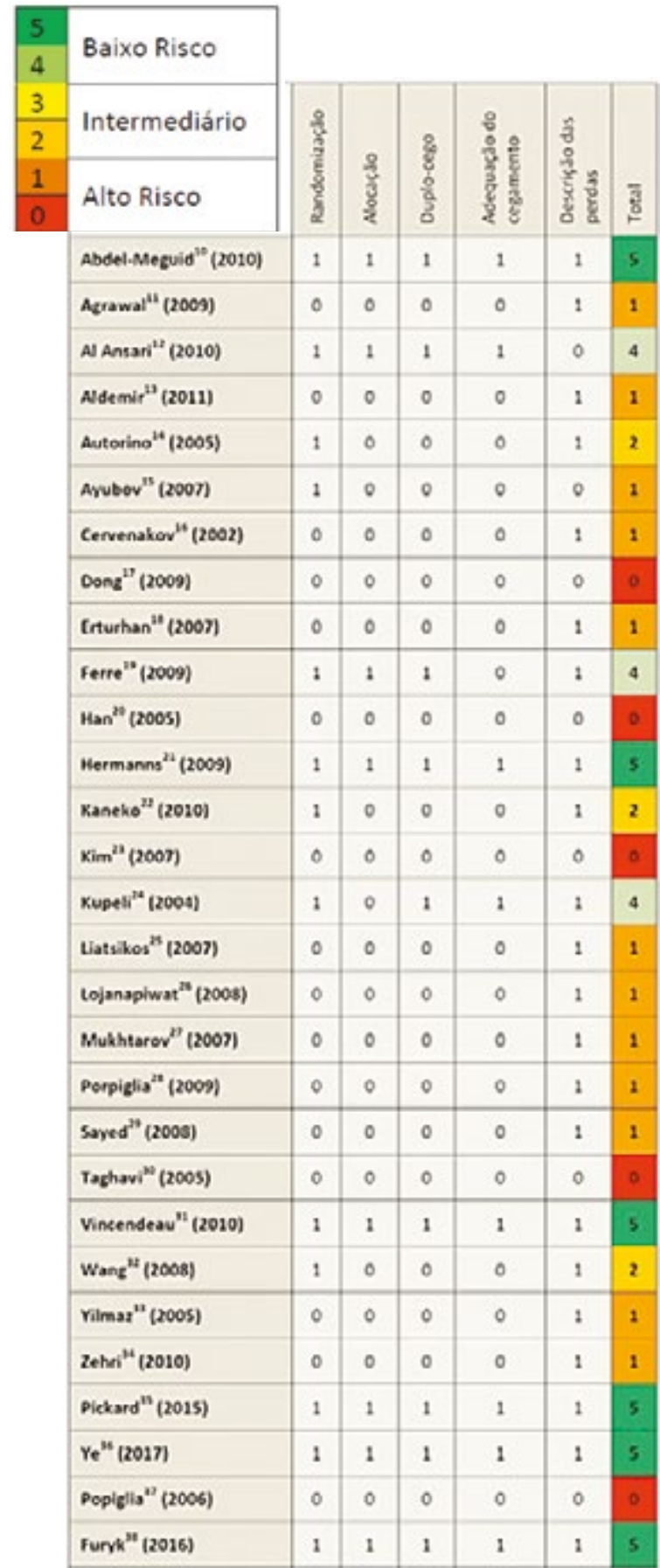

Figura 2 - Classificação dos estudos para risco de viés (Jadad 1996 - Riskof Bias).

\section{Desfechos}

Taxa de Eliminação (ver Figura 3)

O uso de alfa-bloqueador (tansulosina ou doxazosina) aumentou significativamente a taxa de eliminação de cálculos distais quando comparado ao controle $(\mathrm{OR}=3,05, \mathrm{IC} 95 \% 2,38-3,91, p<0,0001)$. Não foi possivel descartar a possibilidade de viés de publicação.

Em relação ao tempo levado para eliminação da pedra (dias a partir do inicio do tratamento), houve diferença significaliva entre os grupos, a favor do alla-bloquea dor ( $M D=-2,32$; IC95\% -3,40 a -1,24; $p<0,0001)$.
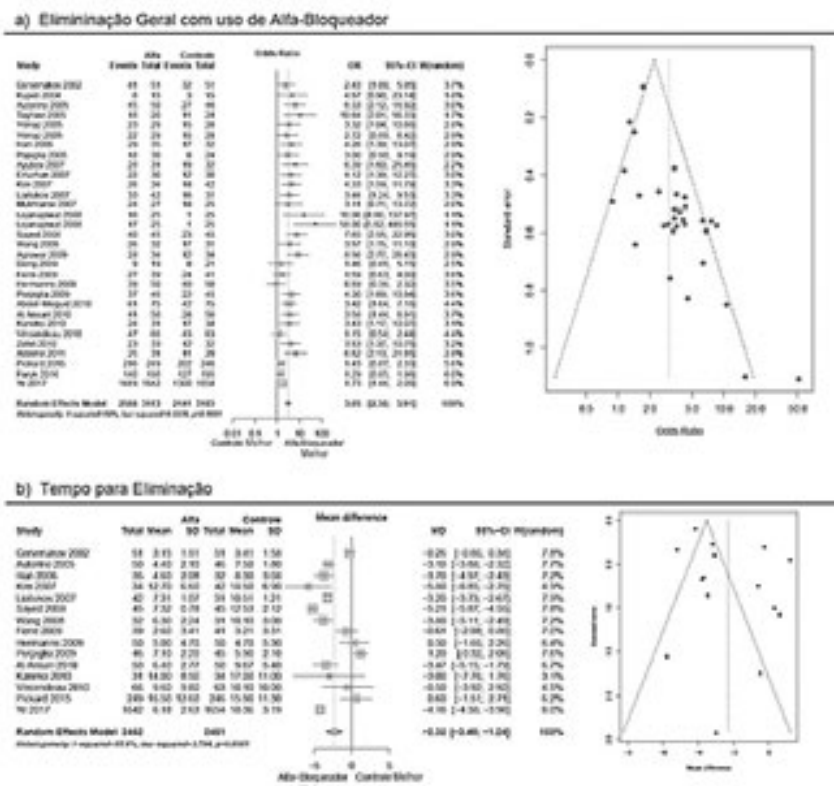

Figura 3 - Efeito dos alfa-bloqueadores na taxa geral de eliminacão de calculos ureterais distais $<10 \mathrm{~mm}$ em relação ao tratamento padrão e/ou placebo.

b) Taxa de Eliminação por Droga (ver Figura 4) Anâlise de estrallicada por droga mostrou efeito positivo na taxa de eliminação de cálculo distal para Tansulosina, tanto na dose de 0,2mg/dia (RR=1,72; IC95\% $1,38$ a 2,$13 ; p<0,0001)$ quanto na dose de $0,4 \mathrm{mg} / \mathrm{dia}$ $(R R=1,35 ;$ IC95\% 1,22 a 1,$49 ; p<0,0001$.

Efeito positivo tambem foi encontrado para Doxazosina quando comparado ao grupo-controle ( $\mathrm{RR}=1,48$; IC95\% 1,26 a 1,75; $p<0,001)$. Apenas para Tansulosina $0,4 \mathrm{mg}$ não foi possível afastar a possibilidade de viés de publicação.
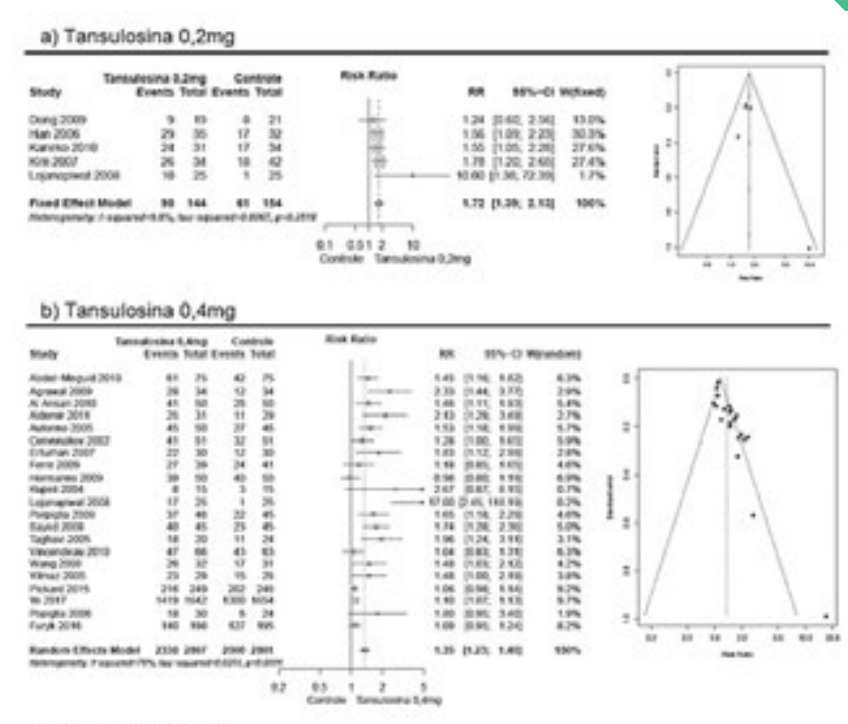

c) Dexazosina 2-4mg

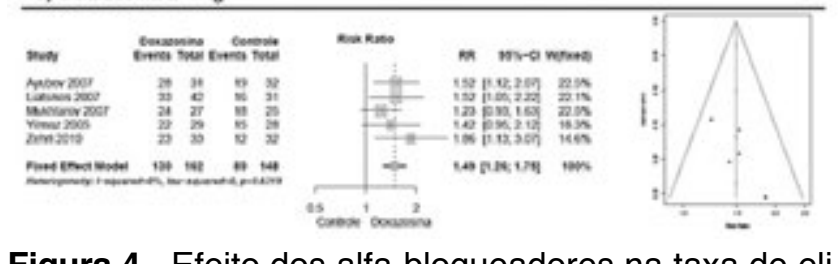

Figura 4 - Efeito dos alfa-bloqueadores na taxa de ellcálculos ureterais distais $<10 \mathrm{~mm}$ estratificado por droga.

c) Taxa de Eliminação por Tamanho (ver Figura 5)

Para cálculos $<5 \mathrm{~mm}$, o uso de alfa-bloqueador não alcançou efeito significativo em relação ao tratamento padrão ou placebo (RR=1, 14; IC $95 \%$ 0,99 a 1,31; $\mathrm{p}=0,0505$ ). Entretanto, para cálculos $>5 \mathrm{~mm}$ houve efeito significativo na taxa de ellmicaça como uso de alfa-bloqueadores ( $\mathrm{OR}=2,33$; IC95\% 1,92 a 2,83; p<0,0001), porém, não fo possível afastar a possibilidade de viés de publicação.

a) cateculos < $5 \mathrm{~mm}$

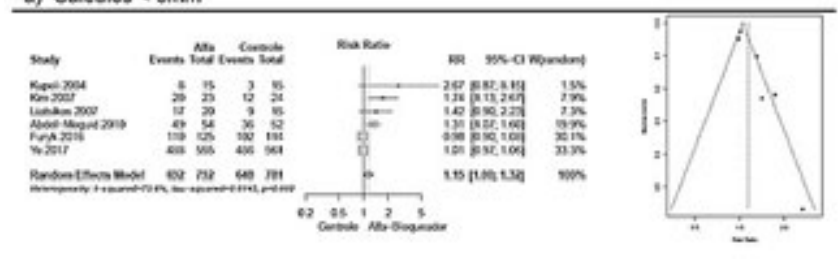
b) Cilculos $\geq 5 \mathrm{~mm}$

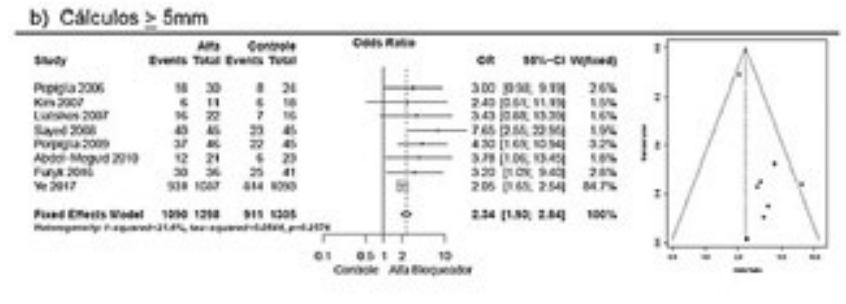

Figura 5 - Efeito dos alfa-bloqueadores na taxa de eliminação de cálculo ureteral distal $<10 \mathrm{~mm}$ estratificado por tamanho da pedra. 
d) Analgesia e Efeitos Adversos (ver Figura 6) A necessidade de analgesia (medida como a dose média de diclofenaco tomado por dia) foi menor no grupo alfa-bloqueador ( $M D=-64,30$; IC95\% -122,09 a $6,51 ; p=0,029)$

Não houve diferença estatisticamente significativa entre os grupos em relação ao número de efeitos colaterais apresentados (RR= $1,27,1 C 95 \% 0,98$ a 1,$66 ; p=$ $0,065)$
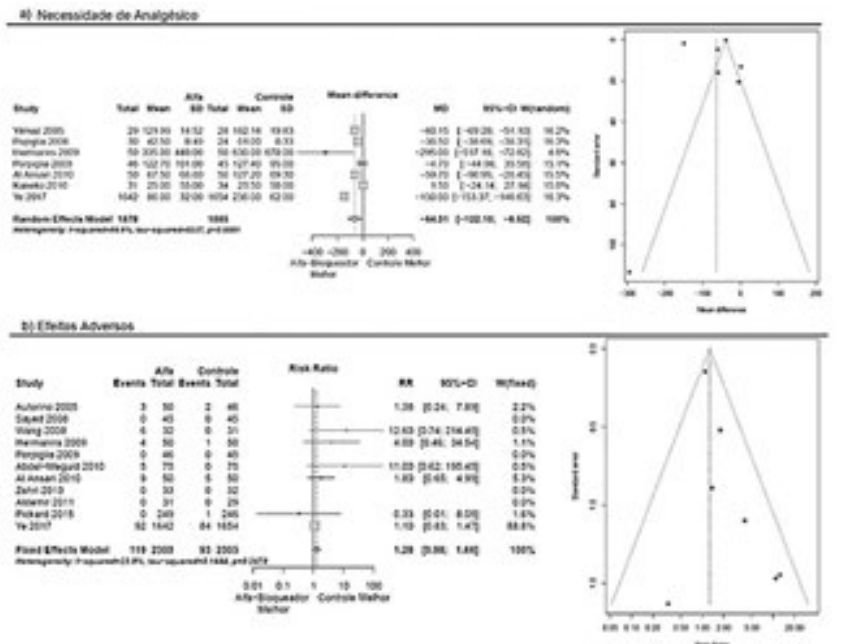

Figura 6 - Comparação entre os grupos alfa-bloqueador e controle para necessidade de analgesia (dose diária de diclofenaco) e para o número de efeitos adversos apresentados em até 4 semanas de seguimento.

\section{DISCUSSÃO}

Esse estudo apresenta uma revisão sistemática dos dados referentes à eficácia dos alfa-bloqueadores no tratamento da ureterolitíase distal para cálculos até $10 \mathrm{~mm}$. Até onde sabemos, é o único dos últimos 4 anos que adicionou os últimos três grandes estudos sobre o tema ao corpo da totalidade das evidências compiladas até então.

Segundo estudo de Pearle (2005), o custo geral do tratamento de cálculos urinários aumentou grandemente nos últimos anos, principalmente devido ao surgimento de novas tecnologias ${ }^{39}$, devendo ser considerado no momento da tomada de decisão terapêutica. Dessa forma, as grandes vantagens do uso da terapia expulsiva no tratamento da ureterolitíase compreendem, entre outras, a não invasividade da terapêutica, o baixo potencial de efeitos adversos e a redução de custos e complicações relacionadas à intervenção cirúrgica e internamento.
Ao bloquear os receptores $a$-adrenérgicos presenes na bainha de Waldeyer do trígono vesical e uréte distal, principalmente os receptores $a-1$, os alfa-bloqueadores propiciam relaxamento da musculatura lisa trigonal e reduzem as pressoes intraureteral pielica retrogradamente ${ }^{40,41}$. Os efeitos são melhor da dor com redução da necessidade de analgésicos facilitação da passagem de cálculos, como demonsrados nesta revisão.

Resultado interessante, já sugerido por ensaios anteriores $\mathrm{s}^{36}$, e corroborado por esta meta-análise, está na interação entre o efeito do alfa-bloqueador e o tamanho do cálculo distal. Não parece haver efeito clinicamente relevante sobre a eliminação de cálculos distais pequenos $(<5 \mathrm{~mm})$, esses teriam grande probabilidade de expulsão independentemente da TME. Do contrário, para os cálculos $>5 \mathrm{~mm}$, o alfa-bloqueador mais que dobrou a chance de expulsão, além de acelerar a eliminação com cerca de 3 dias de diferença. O tamanho de efeito aparenta ser semehante entre Tansulosina e Doxazosina.

Análise de sensibilidade, não apresentada nos resultados, mostra que o escopo das evidências an teriores já sugeria que há um real benefício do uso dessas medicações no tratamento do cálculo ureteral distal apresentado na sala de emergência. Entretanto, até 2008, com base em estudo observacional com medicos americanos, Bandi et. al. concluíram que somente 32\% dos urologistas recomendavam TME para cálculos ureterais distais

Após a revisão de 2014, da colaboração Cochrane com 32 estudos, a TME aparentemente se popularizou e vinha sendo aceita por urologista pacientes até que novos questionamentos foram levantados depois da publicação de dois importantes estudos negativos, o estudo de Pickard (2005), que ficou conhecido como SUSPEND Trial ${ }^{35}$, e o estudo de Meltzer $(2017)^{43}$

O SUSPEND Trial ${ }^{35}$, incluído nesta meta-análise, foi um estudo britanico que, alem de cálculos distais, incluiu cálculos proximais e de uréter médio (Tabela 1), o que pode ter contribuido negativamente para resultado. Alem disso, no estudo, os autores analisaram as taxas globais de eliminação apenas por região ureteral, não tendo sido feita analise estratificada por tamanho, isto é, separadamente para cálculos $>5 \mathrm{~mm}$ e distais, situação na qual o alfa-bloqueador parece guardar seu maior benefício.

Em outro estudo, Meltzer et. al. 2017, randomizaram 512 pacientes adultos com cálculo em média de 3,8mm (1 a 8mm) para Tansulosina ou Placebo e não encontraram diferenca na taxa global de eliminacão de cálculos ${ }^{43}$. Entretanto, houve muita heterogeneidade na seleção dos pacientes com $30 \%$ da amostra contendo cálculos médios, proximais e piélicos, que, somada ao tamanho médio $(3,8 \mathrm{~mm})$ geral das pedras, pode explicar a ausência aparente de efeito da terapia.

O último ensaio publicado, e o maior de todos até o momento, é o estudo de Ye et. al., 2017, incluído nesta meta-analise, que analisou os dados prospectivos de 3.296 pacientes. Houve benefício apenas no subgrupo de indivíduos com cálculos $>5 \mathrm{~mm}$, aumentando a taxa historica de eliminação em 12\% (de 75 para $87 \%)^{36}$. Este parece ser 0 efeito real do alfa-bloqueador, diferença de $12 \%$ em relação ao placebo, 0 que equivale a um NNT de 8,3.

A importância desta revisão está justamente na compilação quantitativa dos dados de todos esses ensaios (exceto o de Meltzer, por não ter preenchido os critérios de inclusão) e na demonstração de efeito benefico dos alfa-bloqueadores no tratamento do cálculo ureteral distal com seu potencial benefício em termos de redução de procedimentos cirúrgicos e gastos com materiais, tecnologia e internação.

Entretanto, existem limitações deste trabalho na generalização dos seus resultados. Não foi possível descartar a presença de viés de publicação para algumas análises e houve importante heterogeneidade entre os estudos, embora neste último caso tenha sido considerada análise sumária com modelo de efeitos randômicos. Essa revisão também não inclui dados de populações mais específicas, como pacientes com comorbidades importantes e grávidas (excluídos dos estudos), além da população pediátrica.

Outros alfa-bloqueadores, como a silodozina e o naftopidil, têm sido investigados para potencial uso na TME com bons resultados ${ }^{44}$, o que chama atenção para um efeito de classe. Em adição, alguns estudos com bloqueadores de canais de cálcio (nefedipina) também têm mostrado bons resultados ${ }^{45}$, o que nos faz pensar a respeito da associação dessas classes no futuro da TME.

Considerando todo o escopo da evidência atual, propomos um novo fluxograma de tomada de decisão frente a um cálculo ureteral distal na sala de emergência. Para cada etapa, o paciente deve tomar parte dos potenciais riscos e benefícios e deve participar ativamente da decisão, tornando-se corresponsave pela escolha final (ver figura 7 ).

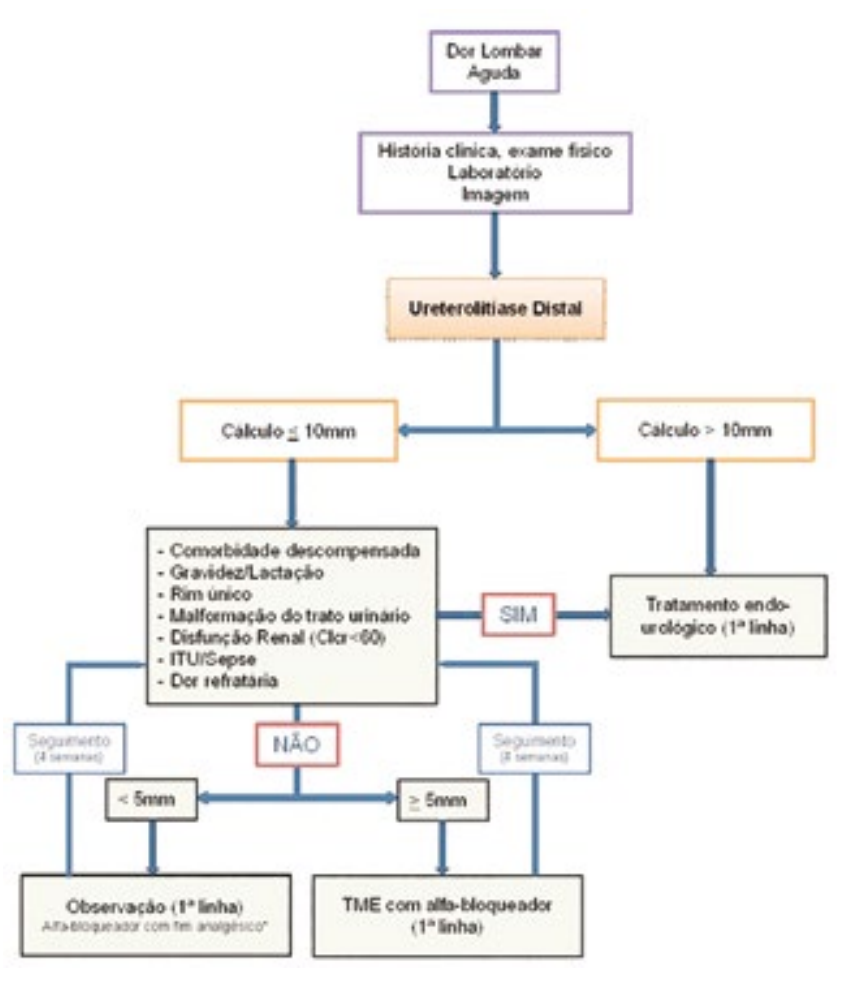

Figura 7 - Fluxograma de tomada de decisão na sala de emergência.

*Os estudos confirmam o efeito analgésico adicional do alfa-bloqueador no cenário de cálculos $<5 \mathrm{~mm}$, no entando, a ausencia de melhoria na taxa de eliminação com potencial aumento do custo total da terapia deve ser considerada.

\section{CONCLUSÃO}

A terapia médica expulsiva com alfa-bloqueador possui efeito geral positivo na taxa de eliminação de cálculos ureterais distais, sabidamente naqueles de $5-10 \mathrm{~mm}$ sem aumentar a incidencia de efeitos colaterais ou de complicaçōes relacionadas. Seu uso, portanto, é benéfico nesse contexto, respeitando-se os critérios de eleição e as contraindicações.

\section{REFERÊNCIAS}

1. Ramello A, Vitale C, Marangella M. Epidemiology of nephrolithiasis. Journal of Nephrology 2000;13 Supp 3: S45-50.

2. Pearle MS, Lotan Y. Urinary lithiasis: etiology, epidemiology, and pathogenesis. In: Campbell MF, Wein AJ, Kavoussi LR editor(s). Campbell-Walsh Urology. 9th Edition. Philadelphia: Saunders Elsevier, 2007.

3. Miller OF, Kane CJ. Time to stone passage for observed ureteral calculi: a guide for patient education. 
Journal of Urology 1999;162(3 Pt 1):688-90.

4. Preminger GM, Tiselius HG, Assimos DG, Alken $P$, Buck AC, Gallucci M, et al.2007 Guideline for the management of ureteral calculi. Journal of Urology 2007;178(6):2418-34

5. Campschroer T, Zhu Y, Duijvesz D, Grobbee DE, Lock MTWT. Alpha-blockers as medical expulsive therapy for ureteral stones. Cochrane Database of Systematic Reviews 2014, Issue 4. Art. No.: CD008509. DOI 10.1002/14651858.CD008509.pub2.

6. Hollingsworth JM, Rogers MAM, Kaufman SR, et al. Medical therapytofacilitateurinarystonepassage: a meta-analysis. Lancet 2006; 368: 1171-79.

7. Moher D, Liberati A, Tetzlaff J, Altman DG, The PRISMA Group (2009). Preferred Reporting Items for Systematic Reviews and Meta-Analyses: The PRISMA (tatement. PLoSMed 6(7): e1000097.

8. Jadad AR, Moore RA, Carroll D, Jenkinson C Reynolds DJ, Gavaghan DJ, et al. Assessingthequalityofreportsofrandomizedclinicaltrials: isblindingnecessary? Control Clin Trials 1996;17(1):1-12.

9. Hozo SP, Djulbegovic B, Hozo I. Estimating the mean and variance from the median, range, and the siz of a sample. BMC Med Res Methodol. 2005;5:13.

10. Abdel-Meguid TA, Tayib A, Al-Sayyad A. Tamsulosin to treat uncomplicated distal ureteral calculi: a double blind randomized placebo-controlled trial. Canadian Journal of Urology 2010 Jun;17(3):5178-83.

11. Agrawal M, Gupta M, Gupta A, Agrawal A, Sarkar A, Lavania P. Prospective randomized trial comparing efficacy of alfuzosin and tamsulosin in management of lower ureteral stones. Urology 2009;73(4):706-9.

12. Al-Ansari A, Al-Naimi A, Alobaidy A, Assadiq K AzmiMD, Shokeir AA. Efficacy of tamsulosin in the management of lower ureteral stones: a randomized double-blind placebocontrolled study of 100 patients. Urology 2010;75(1):4-7.

13. Aldemir M, Ucgul $Y E$, Kayigil O. Evaluation of the efficiency of tamsulosin and Rowatinex in patients with distal ureteral stones: a prospective, randomized, controlled study. International Urology \& Nephrology 2011;43(1): 79-83.

14. Autorino R, De SioM, Damiano R, Di Lorenzo G, Perdona S, Russo A, et al.The use of tamsulosin in the medical treatment of ureteral calculi: where do we stand?. Urological Research 2005;33(6):460-4.

15. Ayubov B, Arustamov D, Mukhtarov S. Efficacy of doxazosin in the management of ureteral stones [abstract no: 368]. European Urology Supplements 2007;6(2):114.

16. Cervenakov I, Fillo J, Mardiak J, Kopecny M, Smi- rala J, Lepies P. Speedy elimination of ureterolithiasis in .

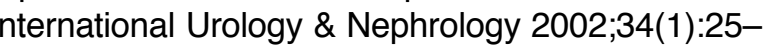
17. Dong IK, Won YC, Tae HK, Jae MC, Park J, Jang HY, et al.Effect of tamsulosin $0.2 \mathrm{mg}$ on the shor-term treatment of urinary stones: multicenter, prospective, randomized stud 2009;50(6):586-90.

18. Erturhan S, Erbagci A, Yagci F, Celik M, Solakhan M, Sarica K. Comparative evaluation of efficacy of use famsulosin and/or tolterodine for medical treatment distal ureteral stones. Urology 2007;69(4):633-6.

19. Ferre RM, Wasielewski JN, Strout TD, Perron AD. Tamsulosin for ureteral stones in theemergencyde AD. Trent: a randomized, controlledtrial. Annals of EmergencyMedicine 2009;54(3):432-9.

20. Han MC, Park YY, Shim BS. Effect of tamsulosin on theexpectant treatment of lower ureteral stones. Korean Journal of Urology 2006;47(7):708-11.

21. Hermanns T, Sauermann P, Rufibach K, Frauenfelder T, Sulser T, Strebel RT. Is there a role for tamsulo$\mathrm{sin}$ in the treatment of distal ureteral stones of $7 \mathrm{~mm}$ or less? Results of a randomised, double-blind, placebocontrolled trial. European Urology 2009;56(3):407-12.

22. Kaneko T, Matsushima H, Morimoto H, Tsuzaka Y, Homma Y. Efficacy of low dose tamsulosin in medica expulsive therapy for ureteral stones in Japanese male patients: a randomized controlled study. International Journal of Urology 2010;17(5):462-5.

23. Kim JW, Cho DY, Lee JG. Effectoftamsulosinontheexpectedtreatmentofupperandlower ureteral stones. Korean JournalofUrology 2007;48(7):724-30.

24. Kupeli B, Irkilata L, Gurocak S, Tunc L, KiracM, Karaoglan U, et al.Doestamsulosinenhancelower ureteral stoneclearancewithorwithoutshockwavelithotripsy? Urology 2004;64(6):1111-5.

25. Liatsikos EN, Katsakiori PF, Assimakopouos $\mathrm{K}$, Voudoukis T, Kallidonis P, Constantinides $\mathrm{C}$, et al.Doxazosin for the management of distal-ureteral stones. JournalofEndourology 2007;21(5):538-41.

26. Lojanapiwat B, Kochakarn W, Suparatchatpan N, Lertwuttichaikul K. Effectivenessoflow-dose andstandarddosetamsulosin in thetreatmentof distal uretericstones: a randomizedcontrolledstudy. Journaloflnternational Medical Research 2008;36(3):529-36.

27. Mukhtarov S, Turdiev A, Fozilov A, Arustamov D, Ayubov $B$. Usingdoxazosin for distal ureteral stoneclearancewithorwithoutshockwavelithotripsy [abstract no: 774]. EuropeanUrologySupplements 2007:6(2):216

28. Porpiglia F, Fiori $\mathrm{C}$, Ghignone G, Vaccino $D$,
Billia M, Morra I, et al.Asecondcycleoftamsulosin in patientswith distal uretericstones: a prospectiverandomizedtrial. BJU International 2009;103(12):1700-3.

29. Sayed MA, Abolyosr A, Abdalla MA, El-Azab AS Efficacy of tamsulosin in medical expulsive therapy for distal ureteral calculi. Scandinavian Journal of Urology \& Nephrology 2008,42(1):59-62.

30. Taghavi R, Darabi MR, Tavakoli K, Keshvari M. Survey of the effect of tamsulosin and nifedipine on facilitating juxtavesical ureteral stone passage [abstract no: 31. Vi. Journal of Endourology 2005, 19(Suppl 1):A9. 31. Vincendeau S, Bellissant E, Houlgatte A, Dore B, Bruyere F, Renault A, et al.Tamsulosin hydrochloride vs placebo for management of distal ureteral stones: a multicentric, randomized, double-blind trial. Archives of

32. Wang CJ, Huang SW, Chang CH. Efficacyofan alpha1 blocker in expulsivetherapyoflower ureteral stones. JournalofEndourology 2008;22(1):41-6.

33. Yilmaz E, Batislam E, Basar MM, Tuglu D, Ferha $\mathrm{M}$, Basar $\mathrm{H}$. The comparisonandefficacyof 3 different alpha1-adrenergic blockers for distal ureteral stones JournalofUrology 2005;173(6):2010-2.

34. Zehri AA, Ather MH, Abbas F, Biyabani SR. Pre liminarystudyofefficacyofdoxazosin as a medical expulsivetherapyof distal uretericstones in a randomizedclinicaltrial. Urology 2010;75(6):1285-8.

35. Pickard R, Starr K, MacLennan G, Lam T, et al. Medical expulsivetherapy in adultswithuretericcolic: a multicentre, randomised, placebo-controlledtrial. Lancet 2015; S0140-6736(15)60933-3, http://dx.doi. org/10.1016/

36. Ye Z, Zeng G, Uang $\mathrm{H}$, Tung $\mathrm{K}$, et al. EfficacyandSafetyofTamsulosin in Medical Expulsive Therapy for Distal Ureteral Stones with Renal Colic: A Multicenter, Randomized, Double-blind, Placebo-controlledTrial. EurUrol (2017), https://doi.org/10.1016/j.eururo.2017.10.033.

37. Popiglia F, Vaccino D, Billia M, Renard J, et al CorticosteroidsandTamsulosin in the Medical ExpulsiveTherapy for Symptomatic Distal Ureter Stones: Single DrugorAssociation? EuropeanUrology 50(2006)339344, doi:10.1016/.eururo.2006.02.023.

38. Furyk J, Chu K, Banks C, Greenslade J, et al. Distal Ureteric Stones andTamsulosin: A Double-Blind, Placebo-Controlled, Randomized, MulticenterTrial. American College of Emergency Physicians 2015. http:// dx.doi.org/10.1016/).annemergmed.2015.06.001.

39. Pearle MS, Calhoun EA, Curhan GC, Urologic Diseases of America Project. Urologic diseases in America project: urolithiasis. Journal of Urology
2005:173(3):848-57.

40. Morita T, Wada I, Saeki H, Tsuchida S,Weis RM. Ureteral urine transport: changes in bolus volume, peristalticfrequency, intraluminalpressureand volum offlowresultingfromautonomicdrugs. Journal of Urology $1987 ; 137(1): 132-5$

41. Sterrett SP, Nakada SY. Medical expulsive therapy. Current Opinion in Urology 2008;18(2):210-3.

42. Bandi G, Best SL, Nakada SY. Current practice patterns in the management of upper urinary tract calculi in the north central Uniled States. Journal of Endourology 2008;22(4):631-6.

43. C. Meltzer, Andrew \& E. Hollander, Judd \& B. Wolfson, Allan \& Kurz, Michael \& V. Jackman, Stephen \& Kirkali, Ziya \& W. Kusek, John \& Burrows, Pamela. (2017). PNFLBA-04 RANDOMIZED CLINICAL TRIAL OF TREATMENT WITH TAMSULOSIN BEGUN IN THE EMERGENCY DEPARTMENT TO PROMOTE PASSAGE OF URINARY STONES. The Journal of Urology. 197. e604. 10.1016/j.juro.2017.03.068.

44. Sun X, He L, Ge W, Lv J. Efficacy of Selective alpha1D-Blocker Naftopidil as Medical Expulsive Therapy for Distal Ureteral Stones. THE JOURNAL OF UROLOGY Vol. 181, 1716-1720, April 2009. DOI:10.1016/. juro.2008.11.118

45. Ye Z, Yang $\mathrm{H}$, Li H, Zhang $\mathrm{X}$, et al. A multicentre, prospective, randomized trial: comparative efficacy of tamsulosin and nifedipine in medical expulsive the rapy for distal ureteric stones with renal colic. JUInernational 2010, (108) 276-279 doi:10.1111/j.1464 410X.2010.09801.x.

1. Serviço de Urologia do HS

E-mail para correspondência:

frarlley@gmail.com 